\title{
POSITIVE FUNCTIONALS AND OSCILLATION CRITERIA FOR SECOND ORDER DIFFERENTIAL SYSTEMS
}

\author{
by GARRET J. ETGEN and ROGER T. LEWIS \\ (Received 30th June 1978)
}

\section{Introduction}

Let $\mathscr{H}$ be a Hilbert space, let $\mathscr{B}=\mathscr{B}(\mathscr{H}, \mathscr{H})$ be the $B^{*}$-algebra of bounded linear operators from $\mathscr{H}$ to $\mathscr{H}$ with the uniform operator topology, and let $\mathscr{S}$ be the subset of $\mathscr{B}$ consisting of the self-adjoint operators. This article is concerned with the second order self-adjoint differential equation

$$
L[Y]=\left[P(x) Y^{\prime}\right]^{\prime}+Q(x) Y=0
$$

on $R^{+}=[0, \infty)$, where $P, Q: R^{+} \rightarrow \mathscr{S}$ are continuous with $P(x)$ positive definite for all $x \in \mathbf{R}^{+}$. Let $\mathscr{G}$ be the set of positive linear functionals on $\mathscr{B}$. Elements of $\mathscr{G}$ are used to derive oscillation criteria for equation (1). It is demonstrated that these criteria include most of the known oscillation criteria for (1) in the matrix and scalar case. Extensions of the results to nonlinear differential equations and to differential inequalities are also discussed.

Appropriate discussions of the concepts of integration and differentiation of $\mathscr{B}$-valued functions, as well as treatments of the existence and uniqueness of solutions $Y: \mathbb{R}^{+} \rightarrow \mathscr{B}$ of (1) can be found in a variety of texts. See, for example, E. Hille (16, Chapters 6 and 9). In particular, it is well known that when suitable initial conditions are specified for (1), then the resulting initial value problem has a unique solution which exists on $\mathbb{R}^{+}$.

In this paper we discuss the behavior of solutions of (1) with particular emphasis on the oscillation of solutions. Studies of the behavior of solutions of second order equations in Banach spaces have been made by several authors, including Hille (16, Chapter 9), T. L. Hayden and H. C. Howard (15), G. J. Etgen and J. F. Pawlowski (10, 11), E. S. Noussair (29), and C. M. Williams (42). The doctoral dissertation of Williams gives a complete treatment of the basic theory of equation (1), and includes existence and uniqueness of solutions, the relationship between (1) and the Riccati equation, nonoscillation, oscillation and disconjugacy.

It is important to note that if $\mathscr{H}=\mathbb{R}_{n}$, Euclidean $n$-space, then $\mathscr{B}$ is the $B^{*}$-algebra of $n \times n$ matrices and equation (1) is the familiar second order self-adjoint matrix differential equation which has been investigated in great detail by a large number of authors. In this regard, we refer to the texts by F. V. Atkinson (4), P. Hartman (14), E. Hille (16), M. Morse (27), W. T. Reid $(31,32)$ and C. A. Swanson (34), as well as to the research papers of C. D. Ahlbrandt, W. Allegretto and L. Erbe, W. A. Coppel, G. J. Etgen, P. Hartman, H. C. Howard, K. Kreith, R. T. Lewis, E. S. Noussair, W. T. Reid, C. A. Swanson, E. C. Tomastik, and V. A. Yakubovic. While there are a variety 
of reasons for considering the behavior of solutions of (1) in either the matrix or general $B^{*}$-algebra case, much of the motivation for such investigations comes from the tremendous amount of research devoted to the second order scalar equation

$$
\left[p(x) y^{\prime}\right]^{\prime}+q(x) y=0
$$

where $p$ and $q$ are continuous, real-valued functions on $\mathrm{R}^{+}$with $p(x)>0$ for all $x \in \mathbf{R}^{+}$. Research on this equation dates to the work of J. Liouville and C. Sturm in the 1830's, and since then, includes the work of authors far too numerous to mention specifically here.

\section{Definitions and Examples}

Throughout this paper we shall assume that $\mathscr{H}$ is a Hilbert space over the reals $\mathbf{R}$, with the inner product on $\mathscr{H}$ denoted by $\langle$,$\rangle and norm \|\|=\langle,\rangle^{1 / 2}$. It will be apparent that the methods and results in the paper apply equally as well when $\mathscr{H}$ is a Hilbert space over the field of complex numbers, but we restrict our attention to real Hilbert spaces because most of the work dealing with the matrix version of (1) and the scalar equation (2) has been done over the reals.

We shall assume that the $B^{*}$-algebra $\mathscr{B}$ of bounded linear operators from $\mathscr{H}$ to $\mathscr{H}$ is topologised by the operator norm

$$
\|A\|=\sup _{\|\alpha\|=1}\|A \alpha\| \text {. }
$$

In the case where $\mathscr{H}=\mathbb{R}_{n}$, the space of ordered $n$-tuples of real numbers, $\mathscr{B}$ is the $B^{*}$-algebra of $n \times n$ matrices with the *-operation being "transpose." The case $\mathscr{H}=\mathbf{R}_{n}$ will be referred to as the finite dimensional case, except when $n=1$. The case $n=1$, i.e., $\mathscr{H}=\mathbb{R}_{1}=\mathbb{R}$ and (1) $\equiv(2)$ will be called the scalar case. The symbol $I$ is used for the identity element of $\mathscr{B}$. The symbol 0 is used indiscriminately for the zero element, with the appropriate interpretation being clear from the context. If $A \in S$, the self-adjoint elements of $\mathscr{B}$, then the notation $A>0(A \geqslant 0)$ is used to signify that $A$ is positive (non-negative) definite.

Let $Y=Y(x)$ be a solution of equation (1). Then it is easy to verify by differentiation that

on $\mathbb{R}^{+}$.

$$
Y^{*}\left[P Y^{\prime}\right]-\left[P Y^{\prime}\right]^{*} Y \equiv C \text { (constant) }
$$

Definition 2.1. A solution $Y=Y(x)$ of equation (1) is conjoined (or prepared) if

$$
Y^{*}\left[P Y^{\prime}\right]-\left[P Y^{\prime}\right]^{*} Y \equiv 0
$$

on $\mathbf{R}^{+}$.

The term "conjoined" has its origins in the Calculus of Variations, and for amplifications of this concept the reader is referred to Morse (27) and to Reid (31). Conjoined solutions of (1) can be obtained simply by choosing conjoined initial values. In fact, it is easy to show that $Y$ is a conjoined solution of (1) if and only if there is at least one point $a \in \mathbf{R}^{+}$such that

$$
Y^{*}(a)\left[P(a) Y^{\prime}(a)\right]=\left[P(a) Y^{\prime}(a)\right]^{*} Y(a) \text {. }
$$


The example given by Noussair and Swanson (28, p. 576) shows that the conjoined hypothesis on the solutions of (1) is needed in order to obtain an analogue of the classical theory of oscillation of the scalar equation (2).

Definition 2.2. A solution $Y=Y(x)$ of equation (1) is nonsingular at $x=a$, $a \in \mathbf{R}^{+}$, if

(i) the range of $Y(a): \mathscr{H} \rightarrow \mathscr{H}$ is $\mathscr{H}$, and

(ii) $Y(a)$ has a bounded inverse.

If either of these conditions fails to hold at $x=a$, then $Y$ is singular at $x=a$. The solution $Y$ has an algebraic singularity at $x=a$ if $Y(a)$ is not one-to-one.

In the finite dimensional case it is clear that the only singularities of a solution $Y$ of (1) are algebraic singularities, and that $Y$ is singular at $x=a$ if and only if det $[Y(a)]=0$. In the general $B^{*}$-algebra case, conditions (i) and (ii) in Definition 2.2 are equivalent to the statement $Y^{-1}(a) \in \mathscr{B}$.

Definitions 2.1 and 2.2 are those used by Hille (16), Hayden and Howard (15), Etgen and Pawlowski (10, 11), and Williams (42). Noussair (29) has introduced slightly different versions of the terms "prepared" and "nonsingular." In particular, he defines:

Definition 2.2'. A solution $Y=Y(x)$ of equation (1) is nonsingular at $x=a$ if $Y(a)$ has a bounded inverse.

Definition 2.1'. A solution $Y=Y(x)$ of equation (1) is prepared if

(i) $Y^{*}\left[P Y^{\prime}\right] \equiv\left[P Y^{\prime}\right]^{*} Y$ on $\mathbb{R}^{+}$,

(ii) there is a constant vector $\alpha \in \mathscr{H}, \alpha \neq 0$, such that $\alpha$ is in the range of $Y(a)$ whenever $Y(a)$ is nonsingular.

Note that in Noussair's definition of nonsingularity at $x=a$, it is not required that $Y(a)$ be onto. An example to illustrate the distinction between Definitions 2.1, 2.2 and Definitions 2.1', 2.2' is given in (29). It will be apparent in the work which follows that the methods of this paper can be applied regardless of which definitions of "conjoined" and "nonsingular" are used. For convenience in the presentation, we shall use Definitions 2.1 and 2.2 throughout the remainder of the paper.

Definition 2.3. A solution $Y=Y(x)$ of equation (1) is nontrivial if there is at least one point $a \in \mathrm{R}^{+}$such that $Y(a)$ is nonsingular.

In the finite dimensional case it is well known that a solution $Y$ of (1) is nontrivial if and only if $Y^{*} Y+\left[P Y^{\prime}\right]^{*}\left[P Y^{\prime}\right]>0$ on $R^{+}$, and that a nontrivial solution has at most a finite number of singular points on any compact subset of $\mathbf{R}^{+}$. These properties do not carry over to the general $B^{*}$-algebra case. Hayden and Howard (15) have shown that while the set of singularities of a nontrivial solution $Y$ of (1) is a closed set, it is possible for the set of singularities to have a finite limit point. It is easy to show that the condition $Y^{*} Y+\left[P Y^{\prime}\right]^{*}\left[P Y^{\prime}\right]>0$ is necessary for $Y$ to be nontrivial, but in the general $B^{*}$-algebra case it is not sufficient as the next example shows.

Example 2.1. Let $\mathscr{H}=l_{2}$, and consider the differential equation

$$
Y^{\prime \prime}+A Y=0 \text { on } \mathrm{R}^{+} \text {, }
$$


where $A$ is the infinite diagonal matrix $A=\pi^{2} \operatorname{diag}[1,1 / 4,1 / 9, \ldots]$. The function $Y: \mathbb{R}^{+} \rightarrow \mathscr{B}$ given by $Y(x)=\operatorname{diag}[\sin \pi x, \sin \pi x / 2, \sin \pi x / 3, \ldots]$ is a solution with the property $Y^{*} Y+Y^{\prime *} Y^{\prime}>0$ on $M^{+}$. However, for each fixed $x \in M^{+}, x$ not an integer, $Y$ is one-to-one but does not have a bounded inverse because because 0 is in the spectrum of $Y(x)$. Clearly $Y$ has an algebraic singularity at each integer $n \in M^{+}$. Thus $Y$ is identically singular on $\mathrm{M}^{+}$.

We turn now to the question of the oscillation of solutions of (1). For the remainder of the paper we shall assume that the term "solution of (1)" means "nontrivial conjoined solution."

Definition 2.4. A solution $Y=Y(x)$ of equation (1) is oscillatory if for each $a \in \mathbb{R}^{+}$there is a number $b, b \geqslant a$, such that $Y(b)$ is singular. The solution $Y$ is non-oscillatory if it is not oscillatory.

In the finite dimensional case a solution $Y$ of (1) is oscillatory if and only if det $Y$ has a infinite number of zeros on $\mathbb{R}^{+}$. Of course, as noted above, det $Y$ can have at most a finite number of zeros on any compact subset of $\mathbb{R}^{+}$. It is a consequence of Morse's generalisation (26) of the Sturm separation theorem that if (1) has an oscillatory solution, then all solutions are oscillatory. The following simple example shows that this property does not carry over to the general $B^{*}$-algebra case.

Example 2.2. Let $P(x) \equiv I$ and $Q(x) \equiv 0$ in (1), i.e., consider the equation $Y^{\prime \prime}=0$ on $\mathbb{R}^{+}$. Every solution $Y$ of the equation has the form $Y(x)=A x+B, A, B \in \mathscr{B}$, and $Y$ is conjoined if $A^{*} B=B^{*} A$. The solution satisfying $Y(0)=I, Y^{\prime}(0)=0$, is $Y(x) \equiv I$. This solution is conjoined and nonsingular on $\mathbb{R}^{+}$. On the other hand, the solution $Z$ given by $Z(x)=A x+I$, where $A=\operatorname{diag}[-1,-1 / 2,-1 / 3, \ldots]$ is conjoined and has an algebraic singularity at each positive integer $n$.

Definition 2.5. Equation (1) is oscillatory if and only if all nontrivial conjoined solutions are oscillatory.

The methods and results of this paper involve the set of positive linear functionals on the Banach algebra $\mathscr{B}$.

Definition 2.6. A linear functional $g: \mathscr{B} \rightarrow \mathbb{R}$ is positive if $g\left(A^{*} A\right) \geqslant 0$ for all $A \in \mathscr{B}$. Equivalently, $g$ is positive if $g(B) \geqslant 0$ whenever $B \in \mathscr{S}$ and $B \geqslant 0$.

Let $\mathscr{G}$ denote the set of positive functionals on $\mathscr{B}$. C. E. Rickart (33) has shown that if $g \in \mathscr{G}$, then $g$ is bounded (i.e., continuous), with $\|g\|=g(I)$, and satisfies a generalised Cauchy-Schwarz inequality

$$
\left[g\left(A^{*} B\right)\right]^{2} \leqslant g\left(A^{*} A\right) g\left(B^{*} B\right)
$$

for all $A, B \in \mathscr{B}$. It follows from (3) that $g$ is the zero functional if and only if $g(I)=0$. If $g$ is not the zero functional, then $g(I)>0$, and, in general, $g(A)>0$ whenever $A>0$.

The set $\mathscr{G}$ is non-empty since it is obvious that the zero functional 0 is an element of $\mathscr{G}$. It is easy to verify, however, that $\mathscr{G}$ contains elements in addition to the zero functional. For example, if $\alpha \in \mathscr{H}, \alpha \neq 0$, then the functional $g_{\alpha}$ defined on $\mathscr{B}$ by

$$
g_{\alpha}(A)=\langle A \alpha, \alpha\rangle
$$


for all $A \in \mathscr{B}$ is a positive functional with $\left\|g_{\alpha}\right\|=g(I)=\|\alpha\|^{2}>0$. Of course the zero functional is "associated" with the zero vector $0 \in \mathscr{H}$ through (4). It can be shown that there are elements in $\mathscr{G}$ which are not "associated" with vectors in $\mathscr{H}$ through (4). For example, if $\mathscr{H}=\mathbf{R}_{n}$, then the functional "trace," denoted $\operatorname{tr}$, and defined by $\operatorname{tr}(A)=$ $\Sigma_{1}^{n} a_{i i}$, is a positive functional which is not the associate of any vector $\alpha \in R_{n}$. In general, it can be verified that $\mathscr{G}$ is a positive cone in the space of continuous linear functionals on $\mathscr{B}$.

Finally, since a positive functional $g$ is continuous, it follows that

$$
g\left[\int_{a}^{x} A(t) d t\right]=\int_{a}^{x} g[A(t)] d t, \quad a, x \in \mathbb{R}^{+}
$$

whenever $A: \mathbf{R}^{+} \rightarrow \mathscr{B}$ is integrable, and

$$
g\left[B^{\prime}(x)\right]=\{g[B(x)]\}^{\prime}
$$

whenever $B: \mathbf{R}^{+} \rightarrow \mathscr{B}$ is differentiable.

\section{Oscillation Criteria}

In this section we develop oscillation criteria for equation (1). These criteria will involve the set $\mathscr{G}$ of positive functionals discussed at the end of the last section. We shall also show how our criteria include a large number of well known oscillation criteria as special cases, and so our approach can be viewed as a unification of the theory. We recall that the term "solution" of (1) is interpreted to mean "nontrivial conjoined solution."

Definition 3.1. A function $V: \mathbb{R}^{+} \rightarrow \mathscr{B}$ is $L$-admissable if each of $V$ and $P V^{\prime}$ is differentiable on $\mathbb{R}^{+}$and

$$
V^{*}\left[P V^{\prime}\right] \equiv\left[P V^{\prime}\right]^{*} V
$$

Our first result is a "Picone type" identity for equation (1). The use of Picone's identity in establishing oscillation and nonoscillation criteria for second order selfadjoint equations is well known. For a complete discussion of the Picone and related identities, the reader is referred to Reid (31, Chapter VII) and to Swanson (36, 37).

Theorem 3.1. Let $g \in \mathscr{G}$, and let $f: \mathbb{R}^{+} \rightarrow \mathbf{R}$ be piecewise continuously differentiable. If $V: \mathbb{R}^{+} \rightarrow \mathscr{B}$ is an L-admissable function which is nonsingular on an interval $J \subseteq \mathbb{R}^{+}$, then

$g\left\{\left(f^{\prime} I-f V^{\prime} V^{-1}\right)^{*} P\left(f^{\prime} I-f V^{\prime} V^{-1}\right)\right\}+\left\{f^{2} g\left[P V^{\prime} V^{-1}\right]\right\}^{\prime}=f^{\prime 2} g[P]-f^{2} g[Q]+f^{2} g\left\{L[V] V^{-1}\right\}$ on $J$.

This identity can be established by a straightforward verification. We use this version of Picone's identity to obtain the following oscillation criterion for equation (1).

Theorem 3.2. If for each $a \in \mathbf{R}^{+}$there is a number $b, b>a$, an element $g \in \mathscr{G}$, $g \neq 0$, and a piecewise continuously differentiable function $f: \mathbf{R}^{+} \rightarrow \mathbf{R}$ such that $f(a)=$ 
$f(b)=0, f \neq 0$ on $[a, b]$, and

$$
\int_{a}^{b}\left\{f^{\prime 2}(x) g[P(x)]-f^{2}(x) g[Q(x)]\right\} d x \leqslant 0,
$$

then equation (1) is oscillatory.

Proof. Suppose there is a solution $V=V(x)$ of (1) such that $V$ is non-oscillatory. Then there is a number $a \in \mathrm{R}^{+}$such that $V$ is nonsingular on $[a, \infty)$.

It is well known that the scalar equation (2) has a solution with at least two zeros on an interval $[a, b]$ if and only if there is a piecewise continuously differentiable function $f: R^{+} \rightarrow \mathbb{R}$ such that $f(a)=f(b)=0, f \not \equiv 0$ on $[a, b]$ and

$$
\int_{a}^{b}\left[f^{\prime 2}(x) p(x)-f^{2}(x) q(x)\right] d x \leqslant 0 .
$$

From this fact we can conclude that there are numbers $c$ and $d, a \leqslant c<d \leqslant b$, and a nontrivial solution $u=u(x)$ of the scalar equation

$$
\left(g[P(x)] y^{\prime}\right)^{\prime}+g[Q(x)] y=0
$$

on $[a, b]$ such that $u(c)=u(d)=0$. From Theorem 3.1 we have that

$$
\begin{aligned}
\int_{c}^{d} g\left\{\left(u^{\prime} I-u V^{\prime} V^{-1}\right)^{*} P\left(u^{\prime} I-u V^{\prime} V^{-1}\right)\right\} d x & =\int_{c}^{d}\left\{u^{\prime 2} g[P]-u^{2} g[Q]\right\} d x \\
& =-\int_{c}^{d}\left\{\left(g[P] u^{\prime}\right)^{\prime}+g[Q] u\right\} u d x \\
& =0 .
\end{aligned}
$$

Now, since $u$ is nontrivial and $u(c)=0$, we must have $u^{\prime}(c) \neq 0$ which implies that there is a nondegenerate interval $\left[c, c^{\prime}\right) \subset[c, d]$ such that $u^{\prime} I-u V^{\prime} V^{-1}$ is nonsingular on $\left[c, c^{\prime}\right)$. Therefore $\left(u^{\prime} I-u V^{\prime} V^{-1}\right)^{*} P\left(u^{\prime} I-u V^{\prime} V^{-1}\right)>0$ on $\left[c, c^{\prime}\right)$ since $P>0$, and this leads to the contradiction

$$
\begin{aligned}
0 & <\int_{c}^{c^{\prime}} g\left\{\left(u^{\prime} I-u V^{\prime} V^{-1}\right)^{*} P\left(u^{\prime} I-u V^{\prime} V^{-1}\right)\right\} d x \\
& \leqslant \int_{c}^{d} g\left\{\left(u^{\prime} I-u V^{\prime} V^{-1}\right)^{*} P\left(u^{\prime} I-u V^{\prime} V^{-1}\right)\right\} d x=0
\end{aligned}
$$
(1).

The proof of Theorem 3.2 suggests the following oscillation criterion for equation

Theorem 3.3. If there is a $g \in \mathscr{G}$ such that the scalar equation

$$
\left(g[P(x)] y^{\prime}\right)^{\prime}+g[Q(x)] y=0
$$

is oscillatory, then equation (1) is oscillatory.

Proof. Simply use an oscillatory solution $u$ of (6) to construct a piecewise continuously differentiable function $f$ such that it, together with the given functional $g \in \mathscr{G}$ satisfies the hypotheses of Theorem 3.2. 
As a consequence of Theorem 3.3, we can consider the question of the oscillation of equation (1) in terms of the oscillation of an associated scalar equation of the form (2). Thus any of the very large number of well known oscillation criteria for (2) can be used to determine a corresponding oscillation criterion for (1). The following corollary is a generalisation of the Leighton-Wintner oscillation criterion, and it is a simple example of the type of criteria which can be obtained for (1) through Theorem 3.3.

Corollary. If there is a $g \in \mathscr{G}$ such that

$$
\int_{0}^{\infty} \frac{d x}{g[P(x)]}=\int_{0}^{\infty} g[Q(x)] d x=\infty
$$

the equation (1) is oscillatory.

Included in this Corollary are most of the well known oscillation criteria for (1) in both the finite and the infinite dimensional cases. We demonstrate this statement by giving some specific examples.

Example 3.1. (Hayden and Howard (15, Theorem 2) and Howard (18, Theorem 1). In equation (1) let $P(x) \equiv I$, and let $K(x)=\int_{0}^{x} Q(t) d t$. If

then equation (1) is oscillatory.

$$
\inf _{\|\alpha\|=1}\langle K(x) \alpha, \alpha\rangle \rightarrow \infty \text { as } x \rightarrow \infty \text {, }
$$

Proof. Fix any $\beta \in \mathscr{H},\|\beta\|=1$, and let $g_{\beta}$ be the positive functional associated with $\beta$ using (4). Then $g_{\beta}(P)=g_{\beta}(I)=\|\beta\|^{2}=1$, and

$$
\inf _{\|\alpha\|=1}\langle K(x) \alpha, \alpha\rangle \leqslant g_{\beta}[K(x)]=\int_{0}^{x} g_{\beta}[Q(t)] d t .
$$

Thus the given hypotheses imply that $\int_{0}^{\infty} 1 / g_{\beta}(P)=\int_{0}^{\infty} g_{\beta}(Q)=\infty$, and the result follows from the Corollary.

Example 3.2. (W. Allegretto and L. Erbe (3, Corollary 1), and Noussair and Swanson (28, Theorem 2)). Let $\mathscr{H}=\mathbf{R}_{n}$. Let $S_{k, n}$ denote the collection of strictly increasing sequences of $k$ integers chosen from the set $\{1, \ldots, n\}$. For any $n \times n$ matrix $A$, and any $\sigma(k)=\left\{i_{1}, i_{2}, \ldots, i_{k}\right\} \in S_{k, n}$, let $\Sigma_{\sigma} A$ denote the sum of the entries of the $k \times k$ submatrix of $A$ obtained by deleting all rows and columns of $A$ except for the $i_{1}, i_{2}, \ldots, i_{k}$ rows and columns. If there exists $\sigma(k) \in S_{k, n}$ such that

$$
\int_{0}^{\infty}\left[\sum_{\sigma} P(x)\right]^{-1} d x=\int_{0}^{\infty}\left[\sum_{\sigma} Q(x)\right] d x=\infty,
$$

then equation (1) is oscillatory. (Noussair and Swanson's result is the case $k=1$ ).

Proof. Let $\alpha$ be the vector with "ones" in the $i_{1}, i_{2}, \ldots, i_{k}$ positions and "zeros" elsewhere, and let $g_{\alpha}$ be the positive functional associated with $\alpha$ using (4). Then $g_{\alpha}(A)=\Sigma_{\sigma} A$ for all $n \times n$ matrices $A$. Thus the hypotheses can be restated as $\int_{0}^{\infty} 1 / g_{\alpha}(P)=\int_{0}^{\infty} g_{\alpha}(Q)=\infty$, and the Corollary applies. 
In a similar manner, the oscillation criteria obtained by such authors as Etgen (9), Kartsatos (19), Kreith (20), Swanson (35), and Tomastik (39) can be demonstrated to be special cases of the Corollary by making suitable choices for the positive functional $g$.

As suggested by Examples 3.1 and 3.2, and by the results in the other papers cited above, most of the oscillation criteria for equation (1) in the finite and infinite dimensional cases are generalisations of the Leighton-Wintner oscillation criterion for the scalar equation (2), i.e., most of the oscillation criteria involve assumptions of the form (7). In contrast, Theorem 3.3 can also be' used to obtain oscillation criteria for equation (1) of the Hille-Wintner type where it is assumed that " $\int_{0}^{\infty} Q(x) d x$ is convergent." As a simple example of this type of criteria, we have:

Example 3.3. Let $P(x) \equiv I$ on $\mathbf{R}^{+}$. If there exists a $g \in \mathscr{G}$ such that $\int_{0}^{\infty} g[Q(x)] d x$ converges (possibly only conditionally), and if

$$
\lim _{x \rightarrow \infty} \inf x \int_{x}^{\infty} g[Q(t)] d t>\frac{1}{4}
$$

then equation (1) is oscillatory.

Our next theorem is an extension of Theorem 3.2, and it is the principal result of this paper. The motivation for this result and the method of proof are contained in the work of D. B. Hinton (17).

Theorem 3.4. If there is a positive continuous function $h$ on $\mathbf{R}^{+}$such that for each $a \in \mathbb{R}^{+}$

$$
\begin{gathered}
\int_{a}^{\infty} h(x) d x=\infty, \\
\lim _{k \rightarrow \infty} \frac{\int_{a}^{t_{k}}\left\{h^{2}(x) g_{k}[P(x)]-\left[\int_{x}^{t_{k}} h(s) d s\right]^{2} g_{k}[Q(x)]\right\} d x}{\left[\int_{a}^{t_{k}} h(x) d x\right]^{2}}=-\infty
\end{gathered}
$$

for some sequence $\left\{t_{k}\right\}$ in $\mathbf{R}^{+}$with $\lim _{k \rightarrow \infty} t_{k}=\infty$, and some sequence of positive functionals $\left\{g_{k}\right\}$ in $\mathscr{G}$ with the property that there exists a positive number $M$ such that $\left\|g_{k}\right\| \leqslant M$ for all positive integers $k$, then equation (1) is oscillatory.

Proof. Choose any $a \in \mathbf{R}^{+}$. For each $t>a$, let $z_{t}:[a, \infty) \rightarrow \mathbf{R}$ be the function defined by

$$
z_{t}= \begin{cases}(x-a) C_{t} & \text { for } x \in[a, a+1) \\ {\left[\int_{x}^{t} h(s) d s\right] /\left[\int_{a}^{t} h(s) d s\right]} & \text { for } x \in[a+1, \infty),\end{cases}
$$

where $C_{t}=\left[\int_{a+1}^{t} h(s) d s\right] /\left[\int_{a}^{t} h(s) d s\right]$. The function $z_{t}$ is piecewise continuously differentiable on $[a, \infty)$.

For any $g \in \mathscr{G}$ and any number $t, t>a+1$, we have

$$
\int_{a}^{t}\left\{\left[z_{t}^{\prime}(x)\right]^{2} g[P(x)]-z_{t}^{2}(x) g[Q(x)]\right\} d x=I_{1}(t)+I_{2}(t)-I_{3}(T),
$$


where

and

$$
\begin{aligned}
& I_{1}(t)=\int_{a}^{a+1}\left\{C_{t}^{2} g[P(x)]-(x-a)^{2} C_{i}^{2} g[Q(x)]\right\} d x, \\
& I_{2}(t)=\frac{\int_{a}^{t}\left\{h^{2}(x) g[P(x)]-\left[\int_{x}^{t} h(s) d s\right]^{2} g[Q(x)]\right\} d x}{\left[\int_{a}^{t} h(s) d s\right]^{2}},
\end{aligned}
$$

$$
I_{3}(t)=\frac{\int_{a}^{a+1}\left\{h^{2}(x) g[P(x)]-\left[\int_{x}^{t} h(s) d s\right]^{2} g[Q(x)]\right\} d x}{\left[\int_{a}^{t} h(s) d s\right]^{2}}
$$

Now $\lim _{t \rightarrow \infty} C_{t}=1$, and so $\lim _{t \rightarrow \infty} \sup \left|I_{1}(t)\right|<\infty$. Also, for $t \in[a, \infty)$,

$$
\begin{aligned}
\left|I_{3}(t)\right| & \leqslant \frac{\int_{a}^{a+1} h^{2}(x) g[P(x)] d x+\int_{a}^{a+1}\left[\int_{x}^{t} h(s) d s\right]^{2}\|g\| \cdot\|Q(x)\| d x}{\left[\int_{a}^{t} h(s) d s\right]^{2}} \\
& \leqslant \frac{\int_{a}^{a+1} h^{2}(x) g[P(x)] d x}{\left[\int_{a}^{t} h(s) d s\right]^{2}}+\frac{\int_{a}^{a+1}\left[\int_{a}^{t} h(s) d s\right]^{2}\|g\| \cdot\|Q(x)\| d x}{\left[\int_{a}^{t} h(s) d s\right]^{2}}
\end{aligned}
$$

so that $\lim _{t \rightarrow \infty} \sup \left|I_{3}(t)\right| \leqslant\|g\| \cdot \max \{\|Q(x)\|: x \in[a, a+1]\}<\infty$. Therefore, by hypothesis, there is a positive integer $k$ such that for $t=t_{k}$ and $g=g_{k}$ we have

$$
\int_{a}^{t_{k}}\left\{\left[z_{t_{k}}^{\prime}(x)\right]^{2} g_{k}[P(x)]-z_{t_{k}}^{2}(x) g_{k}[Q(x)]\right\} d x<0
$$

We can now conclude from Theorem 3.2 that equation (1) is oscillatory.

We conclude this section by giving a variety of oscillation criteria for equation (1) which are corollaries of Theorem 3.4. Each corollary is obtained either by making a particular choice for the sequence $\left\{g_{k}\right\}$ of positive functionals, or a particular choice of the function $h$, as specified in the hypothesis of Theorem 3.4.

Corollary 1. If there is a positive continuous function $h$ on $\mathbb{R}^{+}$such that for each $a \in \mathbb{R}^{+}$

$$
\begin{gathered}
\int_{a}^{\infty} h(x) d x=\infty, \\
\lim _{k \rightarrow \infty} \frac{\int_{a}^{t_{k}}\left\{h^{2}(x)\left\langle P(x) \xi_{k}, \xi_{k}\right\rangle-\left[\int_{x}^{t_{k}} h(s) d s\right]^{2}\left\langle Q(x) \xi_{k}, \xi_{k}\right)\right\} d x}{\left[\int_{a}^{t_{k}} h(s) d s\right]^{2}}=-\infty
\end{gathered}
$$

for some sequence $\left\{t_{k}\right\}$ in $R^{+}$with $\lim _{k \rightarrow \infty} t_{k}=\infty$, and some sequence $\left\{\xi_{k}\right\}$ of unit vectors in $\mathscr{H}$, then equation (1) is oscillatory.

Proof. For each positive integer $k$, the unit vector $\xi_{k}$ determines a positive functional $g_{k}$ on $\mathscr{B}$ using (4). Clearly $\left\|g_{k}\right\|=\left\|\xi_{k}\right\|=1$ for all positive integers $k$, and so the hypotheses of the theorem are satisfied.

In essence the next two corollaries involve assumptions on the eigenvalues of certain self-adjoint operators. While these assumptions do not lead to any difficulties in the finite dimensional case since a self-adjoint operator on $\mathscr{H}=\mathbb{R}_{n}$, i.e., an $n \times n$ symmetric matrix, has exactly $n$ real eigenvalues, the situation is not as simple in the general $B^{*}$-algebra case. In particular, it is well known that there are self-adjoint 
operators which do not have any eigenvalues. For $A \in \mathscr{S}$, the self-adjoint elements of $\mathscr{B}$, let

$$
\mu(A)=\inf _{\|\alpha\|=1}\langle A \alpha, \alpha\rangle \text { and } \sigma(A)=\sup _{\|\alpha\|=1}\langle A \alpha, \alpha\rangle .
$$

Then, for any $A \in \mathscr{S},-\infty<\mu(A) \leqslant \sigma(A)<\infty$, and $\|A\|=\max \{|\mu(A)|,|\sigma(A)|\}$. Moreover, the numbers $\mu(A)$ and $\sigma(A)$ are elements of the spectrum of $A$. In the finite dimensional case, $\mu(A)$ is the minimum eigenvalue of $A$ and $\sigma(A)$ is the maximum eigenvalue of $A$.

Corollary 2. If there is a positive continuous function $h$ on $\mathbf{R}^{+}$such that for each $a \in \mathrm{R}^{+}$

$$
\begin{gathered}
\int_{a}^{\infty} h(x) d x=\infty, \\
\lim _{x \rightarrow \infty} \inf \frac{\mu\left(\int_{a}^{x}\left\{h^{2}(t) P(t)-\left[\int_{t}^{x} h(s) d s\right]^{2} Q(t)\right\} d t\right)}{\left[\int_{a}^{x} h(s) d s\right]^{2}}=-\infty,
\end{gathered}
$$

then equation (1) is oscillatory.

Proof. Let $a \in R^{+}$. Condition (ii) implies that there is a sequence $\left\{t_{k}\right\}$ in $R^{+}$and a corresponding sequence of unit vectors $\left\{\xi_{k}\right\}$ in $\mathscr{H}$ such that

$$
\lim _{k \rightarrow \infty} \frac{\int_{a^{k}}^{t_{k}}\left\{h^{2}(x)\left\langle P(x) \xi_{k}, \xi_{k}\right\rangle-\left[\int_{x}^{t_{k}} h(s) d s\right]^{2}\left\langle Q(x) \xi_{k}, \xi_{k}\right)\right\} d x}{\left[\int_{a}^{t_{k}} h(s) d s\right]^{2}}=-\infty .
$$

Hence, this result follows from Corollary 1.

Corollary 3. If $\int_{0}^{\infty} d x /\|P(x)\|=\infty$, and if for each $a \in \mathbb{R}^{+}$

$$
\lim _{x \rightarrow \infty} \sup \frac{\sigma\left(\int_{a}^{x}\left\{\int_{t}^{x} d s /\|P(s)\|\right\}^{2} Q(t) d t\right)}{\left[\int_{a}^{x} d s /\|P(s)\|\right]^{2}}=\infty,
$$

then equation (1) is oscillatory.

Proof. Define $h$ on $\mathrm{R}^{+}$by $h(x)=1 /\|P(x)\|$. Then by hypothesis, $\int_{a}^{\infty} h(x) d x=\infty$ for each $a \in \mathbf{R}^{+}$. For any $a \in \mathbf{R}^{+}$and any unit vector $\xi$ in $\mathscr{H}$, $\frac{\left\langle\left[\int_{a}^{x}\left\{h^{2}(t) P(t)-\left[\int_{l}^{x} h(s) d s\right]^{2} Q(t)\right\} d t\right] \xi, \xi\right\rangle}{\left[\int_{a}^{x} h(s) d s\right]^{2}} \leqslant$

$$
\frac{1}{\left[\int_{a}^{x} d s /\|P(s)\|\right]}-\frac{\left\langle\left[\int_{a}^{x}\left\{\int_{i}^{x} d s /\|P(s)\|\right\}^{2} Q(t) d t\right] \xi, \xi\right\rangle}{\left[\int_{a}^{x} d s /\|P(s)\|\right]^{2}} .
$$

It is now easy to see that the hypotheses of Corollary 1 are satisfied.

Note that if $P(x) \equiv I$ in equation (1), then the condition

$$
\lim _{x \rightarrow \infty} \sup \frac{1}{(x-a)^{2}} \sigma\left[\int_{a}^{x} \int_{a}^{t} \int_{a}^{s} Q(u) d u d s d t\right]=\infty,
$$

for each $a \in \mathbf{R}^{+}$, implies, by Corollary 3 , that all solutions of $Y^{\prime \prime}+Q(x) Y=0$ are oscillatory. 
Finally, it is important to note that the oscillation criteria of Corollaries 1 through 3 above cannot be obtained as corollaries of Theorem 3.3.

\section{Extensions}

In this section we consider two extensions of the methods and results of the preceding section. The first of these extensions involves the coefficient function $P: \mathrm{R}^{+} \rightarrow \mathscr{S}$ in equation (1). Up to this point we have been assuming that $P(x)>0$ for all $x \in \mathrm{R}^{+}$, but with only minor modifications of the results in Section 3, this requirement can be relaxed to $P(x) \geqslant 0$ for all $x \in \mathbf{R}^{+}$.

The second extension involves a nonlinear analogue of the linear differential operator $L$ in (1). Nonlinear matrix equations, as well as linear and nonlinear matrix differential inequalities, have been investigated by a number of authors. See, for example, Allegretto and Erbe (3), Etgen (7,9), Kreith (20), Noussair and Swanson (28), Swanson (35), and Tomastik (39). An examination of these papers shows that the nonlinear operators are defined in such a manner that the methods developed for linear equations of the form (1) can be applied.

We combine these two extensions into one treatment in the discussion which follows. Since the proofs of the results given in this section are virtually identical to those given in Section 3, most of them will be omitted.

Let $P, Q: \mathbb{R}^{+} \times \mathscr{B}+\mathscr{B} \rightarrow \mathscr{S}$ be continuous with $P(x, A, B) \geqslant 0$ for all $(x, A, B) \in$ $\mathbb{R}^{+} \times \mathscr{B} \times \mathscr{B}$. Let $\Gamma$ denote the collection of functions $Y: \mathbb{R}^{+} \rightarrow \mathscr{B}$ such that $\boldsymbol{Y}$ and $P\left(x, Y, Y^{\prime}\right) Y^{\prime}$ are continuously differentiable and

$$
Y^{*}\left[P\left(x, Y, Y^{\prime}\right) Y^{\prime}\right] \equiv\left[P\left(x, Y, Y^{\prime}\right) Y^{\prime}\right]^{*} Y
$$

on $\mathbb{R}^{+}$. Let $\mathscr{L}$ be the nonlinear differential operator defined on $\Gamma$ by

$$
\mathscr{L}[Y]=\left[P\left(x, Y, Y^{\prime}\right) Y^{\prime}\right]^{\prime}+Q\left(x, Y, Y^{\prime}\right) Y,
$$

and consider the differential inequality

$$
Y^{*} \mathscr{L}[Y] \leqslant 0 .
$$

As an analogue of the work in the preceding sections, we are concerned with the oscillation of solutions of (8). The concepts of oscillation, non-oscillation, etc., of solutions of (8), as well as the oscillation of (8) itself, remain as defined in Section 2. Since the continuation problem is not under consideration here, we assume that all solutions of (8) can be continued over $\mathbf{R}^{+}$.

The first result is the analogue of Theorem 3.1, and is a nonlinear version of Picone's identity.

Theorem 4.1. Let $g \in \mathscr{G}$, and let $f: \mathbf{R}^{+} \rightarrow \mathbf{R}$ be piecewise continuously differentiable. If $V \in \Gamma$ is nonsingular on an interval $J \subseteq \mathrm{R}^{+}$, then

$$
\begin{aligned}
g\left\{[ f ^ { \prime } I - f V ^ { \prime } V ^ { - 1 } ] ^ { * } P ( x , V , V ^ { \prime } ) \left[f^{\prime} I-\right.\right. & \left.\left.f V^{\prime} V^{-1}\right)\right\}+\left\{f^{2} g\left[P\left(x, V, V^{\prime}\right) V^{\prime} V^{-1}\right]\right\}^{\prime} \\
& =f^{\prime 2} g\left[P\left(x, V, V^{\prime}\right)\right]-f^{2} g\left[Q\left(x, V, V^{\prime}\right)\right]+f^{2} g\left\{\mathscr{L}[V] V^{-1}\right\}
\end{aligned}
$$

on $J$. 
Our next result corresponds to Theorem 3.2. The relaxation of the positive definiteness of $P$ on $\mathbf{R}^{+}$will require a strengthening of the inequality corresponding to (5), but, in so doing, the proof is simplified.

Theorem 4.2. The differential inequality (8) is oscillatory if for each $a \in \mathbf{R}^{+}$there is a number $b, b>a$, an element $g \in \mathscr{G}$, and a piecewise continuously differentiable function $f: \mathbf{R}^{+} \rightarrow \mathbf{R}$ such that $f(a)=f(b)=0, f \neq 0$, and

$$
\int_{a}^{b}\left\{f^{\prime 2} g\left[P\left(x, V, V^{\prime}\right)\right]-f^{2} g\left[Q\left(x, V, V^{\prime}\right)\right]\right\} d x<0
$$

for every $V \in \Gamma$ such that $V$ is nonsingular on $[a, \infty)$.

Proof. Suppose there is a solution $V=V(x)$ of $(8), V \in \Gamma$, such that $V$ is nonsingular on $[a, \infty)$ for some $a \in \mathbf{R}^{+}$. Let $f: \mathbf{R}^{+} \rightarrow \mathbf{R}$ and $g \in \mathscr{G}$ be the functions specified in the hypothesis. Then, by integrating (9) from $a$ to $b$, we get

$$
\begin{aligned}
\int_{a}^{b} g\left\{\left[f^{\prime} I-f V^{\prime} V^{-1}\right]^{*} P\left(x, V, V^{\prime}\right)\left[f^{\prime} I-f V^{\prime} V^{-1}\right]\right\} d x \\
=\int_{a}^{b}\left\{f ^ { \prime 2 } g \left[P\left(x, V, V^{\prime}\right]-f^{2} g\left[Q\left(x, V, V^{\prime}\right]\right\} d x+\int_{a}^{b} f^{2} g\left\{\mathscr{L}[V] V^{-1}\right\} d x .\right.\right.
\end{aligned}
$$

Since $P\left(x, V, V^{\prime}\right) \geqslant 0$, the integrand on the left side of this equation is non-negative, and so the number on the left side is non-negative. Since $V$ is a solution of (8), $V^{*} \mathscr{L}[V]=A \leqslant 0$, which implies $\mathscr{L}[V] V^{-1}=V^{*-1} A V^{-1} \leqslant 0$. Therefore the integrand of the second term on the right side of (10) is nonpositive which, with the inequality (9), provides a contradiction.

The final result of this section is a "nonlinear" analogue of Theorem 3.4, and, with obvious modifications, its proof can be used here.

Theorem 4.3. If there is a positive cont inuous function $h$ on $\mathbf{R}^{+}$such that for each $a \in \mathbf{R}^{+}$

$$
\int_{a}^{\infty} h(x) d x=\infty
$$

$$
\lim _{k \rightarrow \infty} \frac{\int_{a}^{t_{k}}\left\{h^{2}(x) g_{k}\left[P\left(x, V, V^{\prime}\right)\right]-\left[\int_{x}^{t_{k}} h(s) d s\right]^{2} g_{k}\left[Q\left(x, V, V^{\prime}\right)\right]\right\} d x}{\left[\int_{a}^{t_{k}} h(s) d s\right]^{2}}=-\infty
$$

for some sequence $\left\{t_{k}\right\}$ in $\mathrm{R}^{+}$with $\lim _{k \rightarrow \infty} t_{k}=\infty$, some sequence of positive functionals $\left\{g_{k}\right\}$ with the property that there exists a positive number $M$ such that $\left\|g_{k}\right\| \leqslant M$ for all positive integers $k$, and for all $V \in \Gamma$ such that $V$ is nonsingular on $[a, \infty)$, then the differential inequality (8) is oscillatory.

In conclusion, we note that Theorem 4.3 has corollaries which correspond in an obvious manner to the corollaries of Theorem 3.3 and 3.4 .

\section{REFERENCES}

(1) C. D. AhLbRANDT, Disconjugacy criteria for self-djoint differential systems, $J$. Differential Equations 6 (1969), 271-295. 
(2) S. AhmAD and A. C. LAZER, Component properties of second order linear systems, Bulletin Amer. Math. Soc. 82 (1979), 287-289.

(3) W. Allegretto and L. ERBE, Oscillation criteria for matrix differential inequalities, Canad. Math. Bull. 16 (1973), 5-10.

(4) F. V. ATKInson, Discrete and Continuous Boundary Problems, (Mathematics in Science and Engineering, Volume 8, Academic Press, New York, 1964).

(5) J. H. BARRETT, Oscillation theory of ordinary linear differential equations, Advances in Mathematics 3 (1969).

(6) W. A. COPPEL, Disconjugacy, (Lecture Notes in Mathematics No. 220, Springer-Verlag, Berlin-Heidelberg-New York, 1971).

(7) G. J. ETGEN, Oscillatory properties of certain nonlinear matrix differential systems, Trans. Amer. Math. Soc. 122 (1966), 289-310.

(8) G. J. ETGEN, Two point boundary problems for second order matrix differential equations, Trans. Amer. Math. Soc. 147 (1970), 119-132.

(9) G. J. ETGEN, Oscillation criteria for nonlinear second order matrix differential equations, Proc. Amer. Math. Soc. 27 (1971), 259-267.

(10) G. J. ETGen and J. F. PAwLowski, Oscillation criteria for second order selfadjoint differential systems, Pacific J. Math. 66 (1976), 99-110.

(11) G. J. ETGEN and J. F. PAWLOWSKI, A comparison theorem and oscillation criteria for second order differential systems, Pacific J. Math. 72 (1977), 59-69.

(12) I. M. Glazman, Direct Methods of Qualitative Spectral Analysis of Singular Differential Operators, (Israel Program for Scientific Translation, Jerusalem, 1965).

(13) P. HARTMAN, Selfadjoint nonoscillatory systems of ordinary second order linear differential equations, Duke Math. J. 24 (1957), 25-35. 1964).

(14) P. HARTMan, Ordinary Differential Equations, (John Wiley and Sons, New York,

(15) T. L. HAYDEN and H. C. HOWARD, Oscillation of differential equations in Banach spaces, Annali di Mathematica Pura ed Applicata 85 (1970), 383-394.

(16) E. Hille, Lectures on Ordinary Differential Equations, (Addison-Wesley Publishing Company, Reading, Massachusetts, 1969).

(17) D. B. Hinton, A criterion for $n-n$ oscillation in differential equations of order $2 n$, Proc. Amer. Math. Soc. 19 (1968), 511-518.

(18) H. C. HowARD, Oscillation criteria for matrix differential equations, Canad. J. Math. 19 (1967), 184-199.

(19) A. G. KARTSATOS, Oscillation of nonlinear systems of matrix differential equations, Proc. Amer. Math. Soc. 30 (1971), 97-101.

(20) K. KREITH, Oscillation criteria for nonlinear matrix differential equations, Proc. Amer. Math. Soc. 26 (1970), 270-272.

(21) K. KREITH, Oscillation Theory, (Lecture Notes in Mathematics, No. 324, SpringerVerlag, Berlin-Heidelberg-New York, 1973).

(22) R. T. LEWIS, Oscillation and nonoscillation criteria for some selfadjoint even order linear differential operators, Pacific J. Math. 51 (1974), 221-234.

(23) R. T. LEWIS, The existence of conjugate points for selfadjoint differential equations of even order, Proc. Amer. Math. Soc. 56 (1976), 162-166. 
(24) R. T. LEWIS, Conjugate points of vector-matrix differential equations, Trans. Amer. Math. Soc., 231 (1977), 167-178.

(25) V. V. MARTYNOV, Conditions for discreteness and continuity of the spectrum in the case of a selfadjoint system of differential equations of even order, Dif. Uravneniya 1 (1965), 1578-1591.

(26) M. MORSE, A generalization of the Sturm separation and comparison theorems in n-space, Math. Ann. 103 (1930), 52-69.

(27) M. Morse, The Calculus of Variations in the Large, (Amer. Math. Soc. Colloq. Publ. 18, Providence, Rhode Island, 1934).

(28) E. S. NousSAIR and C. A. SwANSON, Oscillation criteria for differential systems, $J$. Math. Anal. and Appl. 36 (1971), 575-580.

(29) E. S. NousSAIR, Differential equations in Banach spaces, Bull. Australian Math. Soc. 9 (1973), 219-226.

(30) W. T. REID, Riccati matrix differential equations and nonoscillation criteria for associated linear differential equations, Pacific J. Math. 13 (1963), 665-685.

(31) W. T. REID, Ordinary Differential Equations, (John Wiley and Sons, New York, 1971).

(32) W. T. REID, Riccati Differential Equations, (Mathematics in Science and Engineering, Volume 86, Academic Press, New York, 1972).

(33) C. E. RICKART, General Theory of Banach Algebras, (Van Nostrand, New York, 1960).

(34) C. A. SWANSON, Comparison and Oscillation Theory of Linear Differential Equations, (Mathematics in Science and Engineering, Volume 48, Academic Press, New York, 1968).

(35) C. A. SWANSON, Oscillation criteria for nonlinear matrix differential inequalities, Proc. Amer. Math. Soc. 24 (1970), 824-827.

(36) C. A. Swanson, Remarks on Picone's identities and related identities, Atti. Accad. Naz. Lincer 11 (1972), 1-15.

(37) C. A. Swanson, Picone's identity, Rendiconti di Matematica 8 Serie VI(1975), 373-397.

(38) E. C. TOMASTIK, Singular quadratic functionals of $n$ dependent variables, Trans. Amer. Math. Soc. 124 (1966), 60-76.

(39) E. C. TOMASTIK, Oscillation of nonlinear matrix differential equations of second order, Proc. Amer. Math. Soc. 19 (1968), 1427-1431.

(40) E. C. TOMASTIK, Oscillation of systems of second order differential equations, $J$. Differential Equations 9 (1971), 436-442.

(41) E. C. TOMASTIK, Principal quadratic functionals, Trans. Amer. Math. Soc. 218 (1976), 297-309.

(42) C. M. Williams, Oscillation phenomena for linear differential systems in a $B^{*}$-algebra (Ph.D. Dissertation, University of Oklahoma, 1971).

(43) V. A. YAKUBovic, Oscillation properties of solutions of linear canonical systems of differential equations, Doklady Akad. Nauk. SSR 124 (1959), 533-536.

(44) V. A. YAKUBOVIC, Oscillation and nonoscillation conditions for canonical linear sets of simultaneous differential equations, Doklady Akad. Nauk. SSR 124 (1959), 994-997.

(45) V. A. YAKUBOVIC, Oscillation properties of solutions of canonical equations, Mat. Sb. 56 (1962), 3-42. 